\title{
Structural Covariance in the Human Cortex
}

\author{
Andrea Mechelli, ${ }^{1}$ Karl J. Friston, ${ }^{1}$ Richard S. Frackowiak, ${ }^{1,2}$ and Cathy J. Price ${ }^{1}$ \\ ${ }^{1}$ Functional Imaging Laboratory, Wellcome Department of Imaging Neuroscience, London WC1N 3BG, United Kingdom, and ${ }^{2}$ Istituto di Ricovero e Cura a \\ Carattere Scientifico, Fondazione Santa Lucia, 00179 Rome, Italy
}

The morphology of the human cortex varies remarkably across individuals, regardless of overall brain size. It is currently unclear whether related cortical regions covary in gray matter density, as a result of mutually trophic influences or common experience-related plasticity. We acquired a structural magnetic resonance imaging scan from 172 subjects and extracted the regional gray matter densities from 12 readily identifiable regions of interest involved in sensorimotor or higher-order cognitive functions. We then used these values to predict regional densities in the remaining areas of the cortex, using voxel-based morphometry. This revealed patterns of positive and negative covariance that provide insight into the topographical organization of multiple cortical regions. We report that the gray matter density of a region is a good predictor of the density of the homotopic region in the contralateral hemisphere, with the striking exception of primary visual cortex. Whereas some regions express patterns of regional covariance that are mirror symmetrical relative to the interhemispheric fissure, other regions express asymmetric patterns of regional covariance. Finally, patterns of covariance are remarkably consistent between males and females, with the exception of the left amygdala, which is positively associated with the left and right anterior inferior temporal cortex in males and with the right angular gyrus in females. Our study establishes that the density of different cortical regions is coordinated within an individual. The coordinated variations we report are likely to be determined by both genetic and environmental factors and may be the basis for differences in individual behavior.

Key words: cortex; homotopic; heterotopic; ipsilateral; anatomy; VBM

\section{Introduction}

The morphology of the human cortex differs substantially across individuals. For instance, most regions express remarkable variability that is not a simple function of overall brain size (Kennedy et al., 1998). Little is known, however, about the topographical principles that govern this structural variability across individuals. One possibility is that the density of each region is uniquely determined in each subject, presumably by a combination of genetic and environmental factors. According to this view, the gray matter density in different regions should not covary across individuals once differences attributable to overall brain size have been accounted for. An alternative possibility is that related regions covary in density as a result of mutually trophic influences (Ferrer et al., 1995) or common experience-related plasticity (Draganski et al., 2004; Mechelli et al., 2004). This hypothesis is supported by the observation that related components of the visual system (i.e., optic tract, lateral geniculate nucleus, and primary visual cortex) covary in volume across individuals (Andrews et al., 1997). Furthermore, significant covariance in the regional volume of circumscribed brain regions has been reported in studies of age-related decline (Raz et al., 1997, 2004, 2005).

Received Jan. 26, 2005; revised June 24, 2005; accepted July 18, 2005.

This work was supported by the Wellcome Trust. A.M. was supported by the National Institutes of Health. We thank Joel Winston and Klaas Enno Stephan for helpful discussions.

Correspondence should be addressed to Andrea Mechelli, Wellcome Department of Imaging Neuroscience, 12 Queen Square, London WC1N 3BG, UK. E-mail: andream@fil.ion.ucl.ac.uk.

DOI:10.1523/JNEUROSCI.0357-05.2005

Copyright $\odot 2005$ Society for Neuroscience $\quad$ 0270-6474/05/258303-08\$15.00/0
Here we used voxel-based morphometry (VBM), a wholebrain semiautomated technique for characterizing structural brain differences in vivo (Ashburner and Friston, 2000; Mechelli et al., 2005), to investigate whether there is significant covariance in gray matter density (i.e., cubic millimeters of gray matter per voxel) among different regions of the human cortex. After acquiring a structural magnetic resonance imaging (MRI) scan from 172 subjects, we extracted the local densities from 12 readily identifiable regions of interest involved in sensorimotor or higher-order cognitive functions. We then used these values as "predictors" of regional densities in all other areas of the cortex and examined both positive and negative correlations. We specifically addressed three related questions that are critical for understanding the topographical organization of multiple regions within the human cortex. First, are there regions that positively or negatively covary in density across individuals? We predicted significant structural covariance based on previous reports of positive (Raz et al., 2004, 2005) and negative (Raz et al., 1997, 2005) correlations between regional volumes of circumscribed brain regions. Second, are such patterns of regional covariance mirror symmetrical in the two hemispheres? Previous studies have shown strong lateralization in the human brain at both structural (Good et al., 2001) and functional (Stephan et al., 2003) levels. We therefore hypothesized that such lateralization might also be expressed in terms of asymmetric patterns of regional covariance in the two hemispheres. Third, are patterns of regional covariance consistent between male and female populations? Several studies have reported gender-related differences in brain function, especially in the context of emotionally charged stimuli (Canli et al., 2002). We therefore predicted that any gender- 
related differences in structural covariance would be found in regions that have been consistently reported to express genderrelated activations, such as the amygdala (Cahill et al., 2004). Our results reveal distinct sets of regions that positively or negatively covary in density across individuals and provide insight into the topographical organization of multiple regions within the human cortex.

\section{Materials and Methods}

Subjects. The study was approved by the Joint Ethics Committee of the Institute of Neurology (University College London, London, UK) and National Hospital for Neurology and Neurosurgery (National Health Service Trust, London, UK). Informed consent was obtained from a total of 172 right-handed volunteers with no history of neurological or psychiatric disorders. These included 102 males (aged 18-41 years; mean of 26.8 years) and 70 females (aged $18-40$ years; mean of 26.2 years).

Data acquisition. A structural MRI image was acquired from each subject, using a 2 Tesla Siemens (Erlagen, Germany) MAGNETOM Vision scanner. Scanning parameters were as follows: T1-weighted MPRAGE sequence; flip angle, $12^{\circ}$; matrix size, $256 \times 192$; field of view, $256 \times 192$; yielding 108 sagittal slices, with slice thickness of $1.5 \mathrm{~mm}$ and in-plane resolution of $1 \times 1 \mathrm{~mm}$. The same scanner parameters and scanner hardware were used for the acquisition of all anatomical volumes.

Data analysis. Structural images were preprocessed using VBM implemented with Statistical Parametric Mapping software (SPM2) running under Matlab 6.0 (MathWorks, Natick, MA). VBM is a whole-brain, unbiased, semiautomated technique for characterizing regional cerebral differences in structural magnetic resonance images (Ashburner and Friston, 2000; Mechelli et al., 2005). First, structural images were segmented to extract gray matter and then normalized to an asymmetric T1-weighted template in Montreal Neurological Institute (MNI) stereotactic space, in a recursive manner. Image segmentation incorporated an intensity non-uniformity correction to account for smooth intensity variations caused by gradient distortions and different positions of cranial structures within the MRI coil (Ashburner and Friston, 2000). An additional step was added to ensure that the total amount of gray matter in each voxel was conserved before and after spatial normalization (Good et al., 2001). This "modulation" step involves multiplying the spatially normalized gray matter by its relative volume before and after spatial normalization. The resulting gray matter images were finally smoothed with a $12 \mathrm{~mm}$ isotropic Gaussian kernel. This smoothing kernel was chosen to compensate for the inexact nature of spatial normalization and to maximize the chance that regional effects are expressed at a spatial scale in which homologies in structural anatomy exist over subjects. A 12 $\mathrm{mm}$ Gaussian kernel had proved to be a reasonable filter in previous VBM studies (Good et al., 2001; Sluming et al., 2002; Mechelli et al., 2004). After smoothing, each voxel represents the local average amount of gray or white matter in the region, the size of which is defined by the smoothing kernel.

Statistical analysis was performed on the gray matter images using the general linear model as implemented in SPM2. Regional densities were extracted from 12 regions of interest in the 172 preprocessed gray matter segments. Regions of interest included bilateral pars triangularis in inferior frontal cortex $(x= \pm 59, y=11, z=8)$, primary auditory cortex $(x=$ $\pm 43, y=-24, z=13)$, primary somatosensory cortex (S1) ( $x= \pm 27$, $y=-36, z=60)$, primary visual cortex (V1), $(x= \pm 24, y=-95, z=$ $-6)$, intraparietal sulcus $(x= \pm 24, y=-60, z=63)$, and amygdala $(x=$ $\pm 18, y=0, z=-24)$. These regions were selected because they have been extensively characterized at the functional level by previous neuropsychological and neuroimaging studies. We specifically chose regions that were involved in sensorimotor functions (i.e., primary visual cortex, primary auditory cortex, and S1) or higher-order cognitive processes such as emotion (i.e., amygdala), attention (i.e., intraparietal cortex), and language (i.e., inferior frontal cortex). The choice of these regions was also motivated by the fact that they were characterized by well defined anatomical landmarks and therefore readily identifiable. The exact coordinates were chosen based on a review of the neuroimaging literature and anatomically validated using the standard T1-weighted template in MNI stereotactic space.

The regional densities extracted from our regions of interest were then used to model regional densities in all voxels of the preprocessed gray matter segments. A separate correlation analysis was performed for each region of interest, resulting in 12 statistical analyses. To characterize gender-specific effects, males and females were modeled separately in all analyses (i.e., we modeled gender $\times$ gray matter interactions). In addition, the total amount of gray matter in each image was entered as a confounding variable to identify regionally specific effects that could not be explained by overall gray matter density. To discard any pattern of covariation that could be attributed to region-dependent age effects, age was also entered as a variable of no interest.

One of the aims of the present study was to test for asymmetric patterns of regional covariance in the two hemispheres. This required flipping the left-right orientation of the structural MRI images and then modeling flipped and unflipped scans within the same statistical model. A direct comparison between homotopic regions in flipped and unflipped images was then used to test for the interaction between structural covariance and hemisphere. This laterality analysis was performed for each pair of homotopic regions, resulting in six additional statistical analyses.

Our analyses identified (1) voxels in which density covaried positively with our regions of interest, regardless of gender, (2) voxels in which density covaried negatively with our regions of interest, regardless of gender, (3) voxels that expressed asymmetric patterns of covariance in the two hemispheres, and (4) voxels that expressed differential patterns of covariance in males and females. Statistical inferences were based on $t$ tests and were made at $p<0.05$ (corrected for multiple comparisons across the whole brain).

\section{Results}

We define two regions as being positively associated if increased gray matter in one area is associated with increased gray matter in the other. In contrast, two regions are negatively associated if increased gray matter in one area is associated with reduced gray matter in the other. We refer to homotopic regions to indicate the same areas in opposite hemispheres (e.g., left and right inferior frontal gyri), we refer to ipsilateral regions to indicate different areas in the same hemisphere (e.g., left inferior frontal gyrus and left amygdala), and we refer to heterotopic regions to indicate different areas in opposite hemispheres (e.g., left inferior frontal gyrus and right amygdala). Our investigation revealed positive and negative associations between homotopic regions, between ipsilateral regions, and between heterotopic regions.

\section{Symmetric associations}

We first report patterns of structural associations that were mirror symmetrical relative to the interhemispheric fissure. Positive, symmetric associations were found between homotopic regions, between ipsilateral regions, and between heterotopic regions $(\mathrm{Ta}-$ ble 1, Fig. 1). In contrast, negative symmetric associations were only found between ipsilateral regions and between heterotopic regions (Table 2) (supplemental Fig. S1, available at www. jneurosci.org as supplemental material).

\section{Homotopic effects}

We found that the density of a region in one hemisphere is a good predictor of the density of the homotopic region in the contralateral hemisphere (Fig. 2). For instance, the density of the amygdala in the left hemisphere positively correlates with the density of the amygdala in the right hemisphere and vice versa. Similar homotopic effects were observed in the inferior frontal, primary auditory, primary somatosensory, and intraparietal cortex (Table 2). However, we found one striking exception in the V1. The density of this region in one hemisphere did not covary with the density 
Table 1. Positive, symmetric associations between our regions of interest and the other areas of the human cortex

\begin{tabular}{|c|c|c|c|c|}
\hline & $x$ & $y$ & $z$ & Z-score \\
\hline \multicolumn{5}{|l|}{ Left inferior frontal, pars triangularis } \\
\hline \multicolumn{5}{|l|}{ Homotopic effects } \\
\hline Right inferior frontal, pars triangularis & 59 & 12 & 5 & 7.5 \\
\hline \multicolumn{5}{|l|}{ Ipsilateral effects } \\
\hline Left inferior frontal, pars orbitalis & -51 & 41 & 3 & 6.2 \\
\hline Left postcentral & -65 & -20 & 17 & 4.5 \\
\hline Left anterior superior temporal & -53 & 11 & -23 & 5.3 \\
\hline \multicolumn{5}{|l|}{ Heterotopic effects } \\
\hline Right inferior frontal, pars orbitalis & 51 & 45 & 3 & 5.3 \\
\hline \multicolumn{5}{|l|}{ Right inferior frontal, pars triangularis } \\
\hline \multicolumn{5}{|l|}{ Homotopic effects } \\
\hline Left inferior frontal, pars triangularis & -59 & 8 & 6 & 7.6 \\
\hline \multicolumn{5}{|l|}{ Ipsilateral effects } \\
\hline Right inferior frontal, pars orbitalis & 54 & 39 & 3 & 5.8 \\
\hline Right postcentral & 65 & -17 & 17 & 4.6 \\
\hline Right anterior superior temporal & 57 & 11 & -15 & 5.5 \\
\hline \multicolumn{5}{|l|}{ Heterotopic effects } \\
\hline Left inferior frontal, pars opercularis & 54 & 14 & 21 & 4.6 \\
\hline \multicolumn{5}{|l|}{$\begin{array}{l}\text { Left primary visual cortex } \\
\text { NS }\end{array}$} \\
\hline \multicolumn{5}{|l|}{ Right primary visual cortex } \\
\hline NS & & & & \\
\hline \multicolumn{5}{|l|}{ Left primary auditory cortex } \\
\hline \multicolumn{5}{|l|}{ Homotopic effects } \\
\hline Right primary auditory cortex & 42 & -20 & 14 & 8.3 \\
\hline \multicolumn{5}{|l|}{ Right primary auditory cortex } \\
\hline \multicolumn{5}{|l|}{ Homotopic effects } \\
\hline Left primary auditory cortex & -41 & -23 & 14 & 8.4 \\
\hline Left amygdala & & & & \\
\hline Homotopic effects & & & & \\
\hline Right amygdala & 18 & 2 & -26 & 8.3 \\
\hline Ipsilateral effects & & & & \\
\hline Left temporal pole & -39 & 8 & -39 & 7.5 \\
\hline Heterotopic effects & & & & \\
\hline Right temporal pole & 32 & 14 & -35 & 6.6 \\
\hline Right amygdala & & & & \\
\hline Homotopic effects & & & & \\
\hline Left amygdala & -18 & -3 & -26 & 8.3 \\
\hline Ipsilateral effects & & & & \\
\hline Right temporal pole & 36 & 6 & -42 & 6.7 \\
\hline Heterotopic effects & & & & \\
\hline Left temporal pole & -32 & 9 & -38 & 4.8 \\
\hline Left S1 & & & & \\
\hline Homotopic effects & & & & \\
\hline Right S1 & 27 & -35 & 63 & 7.6 \\
\hline Ipsilateral effects & & & & \\
\hline Left parahippocampal gyrus & -9 & -32 & -5 & 6.4 \\
\hline Left precentral gyrus & -45 & -11 & 47 & 5.5 \\
\hline Left superior frontal gyrus & -11 & -2 & 60 & 5.6 \\
\hline Left anterior cingulate & -6 & 39 & 21 & 5.3 \\
\hline Left middle frontal gyrus & -45 & 11 & 42 & 4.4 \\
\hline Heterotopic effects & & & & \\
\hline Right parahippocampal gyrus & 11 & -32 & -2 & 6.2 \\
\hline Right precentral gyrus & 46 & -12 & 51 & 4.6 \\
\hline Right anterior cingulate & 6 & 45 & 22 & 4.7 \\
\hline Right middle frontal gyrus & 50 & 9 & 41 & 4.7 \\
\hline Right superior frontal gyrus & 5 & -2 & 51 & 4.6 \\
\hline Right S1 & & & & \\
\hline Homotopic effects & & & & \\
\hline Left S1 & -30 & -36 & 62 & 7.6 \\
\hline Ipsilateral effects & & & & \\
\hline Right parahippocampal gyrus & 11 & -33 & -6 & 6.6 \\
\hline Right precentral gyrus & 45 & -12 & 46 & 5.1 \\
\hline Right superior frontal gyrus & 6 & -11 & 59 & 5.1 \\
\hline Right anterior cingulate & 2 & 42 & 23 & 5.0 \\
\hline
\end{tabular}

Table 1. Continued

\begin{tabular}{|c|c|c|c|c|}
\hline & $x$ & $y$ & $z$ & Z-score \\
\hline Right middle frontal gyrus & 48 & 9 & 41 & 4.9 \\
\hline \multicolumn{5}{|l|}{ Heterotopic effects } \\
\hline Left parahippocampal gyrus & -11 & -33 & -6 & 6.7 \\
\hline Left precentral gyrus & -45 & -12 & 47 & 5.9 \\
\hline Left anterior cingulate & -5 & 39 & 21 & 5.0 \\
\hline Left middle frontal gyrus & -47 & 11 & 42 & 4.7 \\
\hline Left superior frontal gyrus & -11 & -2 & 62 & 5.3 \\
\hline \multicolumn{5}{|l|}{ Left intraparietal } \\
\hline \multicolumn{5}{|l|}{ Homotopic effects } \\
\hline \multicolumn{4}{|l|}{ Ipsilateral effects } & 6.3 \\
\hline Left postcentral gyrus & -30 & -40 & 61 & 4.9 \\
\hline \multicolumn{5}{|l|}{ Heterotopic effects } \\
\hline Right postcentral gyrus & 28 & -45 & 66 & 4.6 \\
\hline \multicolumn{5}{|l|}{ Right intraparietal } \\
\hline $\begin{array}{l}\text { Homotopic effects } \\
\text { Left intraparietal } \\
\text { Ipsilateral effects }\end{array}$ & -26 & -56 & 62 & 6.9 \\
\hline Right postcentral gyrus & 30 & -39 & 63 & 6.4 \\
\hline \multicolumn{5}{|l|}{ Heterotopic effects } \\
\hline Left postcentral gyrus & -30 & -39 & 62 & 6.0 \\
\hline
\end{tabular}

AllZ-scores are significant at $p<0.05$ (corrected). These effects are consistent for males (M) and females (F). NS, No significant.

of the same region in the contralateral hemisphere, even when lowering the statistical threshold to $p<0.001$ (uncorrected). We therefore explored the specificity of this dissociation by considering the correlation between left and right extrastriate visual cortices. This revealed a positive association between left and right medial extrastriate visual cortex $(x= \pm 34, y=-90, z=$ -6 ; Z-score $=4.6 ; p<0.05$ corrected) and between left and right lateral extrastriate visual cortex $(x= \pm 40, y=-84, z=-8$; $\mathrm{Z}$-score $=5.8 ; p<0.05$ corrected). A direct statistical comparison between correlation coefficients (using Fisher's $z^{\prime}$ transformation) (Kleinbaum et al., 1988) established that the correlation between left and right V1 was significantly less than the correlations between left and right medial visual cortex $(p<0.001)$ and between left and right lateral visual cortex $(p<0.0001)$. Thus, the dissociation between left and right visual areas appeared to be specific to V1.

Ipsilateral effects

We found that the density of a region in one hemisphere may covary with the density of one or more regions in the same hemisphere. For instance, the density of the amygdala positively correlates with the density of the ipsilateral temporal pole but negatively correlates with the density of the ipsilateral supramarginal gyrus. Ipsilateral effects were also observed in inferior frontal cortex, primary somatosensory cortex, and intraparietal cortex (Tables 1, 2). In contrast, we did not detect any ipsilateral effects in primary visual cortex and primary auditory cortex that survived our statistical threshold ( $p<0.05$ corrected).

\section{Heterotopic effects}

We found that the density of a region in one hemisphere may covary with the density of one or more heterotopic regions in the contralateral hemisphere. For instance, the density of the amygdala positively correlates with the density of the contralateral temporal pole but negatively correlates with the density of the contralateral supramarginal gyrus. Heterotopic effects were also observed in inferior frontal cortex, primary somatosensory cortex, and intraparietal cortex (Tables 1, 2). In contrast, there were no hetero- 
topic effects in primary visual cortex and primary auditory cortex that survived our statistical threshold ( $p<0.05$ corrected).

\section{Asymmetric associations}

We now report homotopic regions that expressed asymmetric patterns of regional covariances. This was assessed by directly comparing structural correlations in flipped and unflipped MRI images within the same statistical model $(p<0.05$ corrected). We detected several asymmetric associations between ipsilateral regions and between heterotopic regions (Table 3 ) (supplemental Fig. S2, available at www. jneurosci.org as supplemental material).

\section{Ipsilateral effects}

Asymmetric ipsilateral effects were observed when examining two of our regions of interest: inferior frontal cortex and primary somatosensory cortex. The inferior frontal cortex expressed stronger association with the pars opercularis in the right relative to the left hemisphere. Inspection of the hemisphere-specific correlations revealed that this asymmetry was attributable to a positive association between inferior frontal cortex and the pars opercularis in the right hemisphere but not in the left hemisphere (for details, see Table 3 ). The primary somatosensory cortex expressed stronger association with superior frontopolar gyrus and cuneus in the right relative to the left hemisphere, and with superior frontal gyrus, parahippocampal gyrus, and paracentral lobule in the left relative to the right hemisphere. The right $>$ left asymmetries were attributable to strong negative associations in the left hemisphere and no effect in the right hemisphere, whereas the left $>$ right asymmetries were attributable to strong positive associations in the left hemisphere and no effect in the right hemisphere (Table 3).

\section{Heterotopic effects}

Asymmetric heterotopic effects were observed when examining two of our regions of interest: primary somatosensory cortex and intraparietal cortex. The association with the contralateral superior parietal gyrus and angular gyrus was stronger in the right than the left primary somatosensory cortex, and the association with the contralateral superior frontal gyrus and cerebellum was stronger in the left than the right primary somatosensory cortex. Inspection of the hemisphere-specific correlations revealed that the right $>$ left asymmetries were attributable to positive associations in the right but not the left hemisphere, whereas the left $>$ right asymmetries were attributable to positive correlations in the left but not the right hemisphere (Table 3). In addition, the association with the contralateral angular gyrus was stronger in the right than the left intraparietal cortex. This asymmetry could be explained by a strong positive association in the right but not the left hemisphere (Table 3).

\section{Gender effects}

The effects reported so far were remarkably consistent for male and female individuals. For instance, all positive and negative associations reported above replicated in the male and female samples separately ( $p<0.001$ uncorrected). However, the left

\section{Positive Associations}
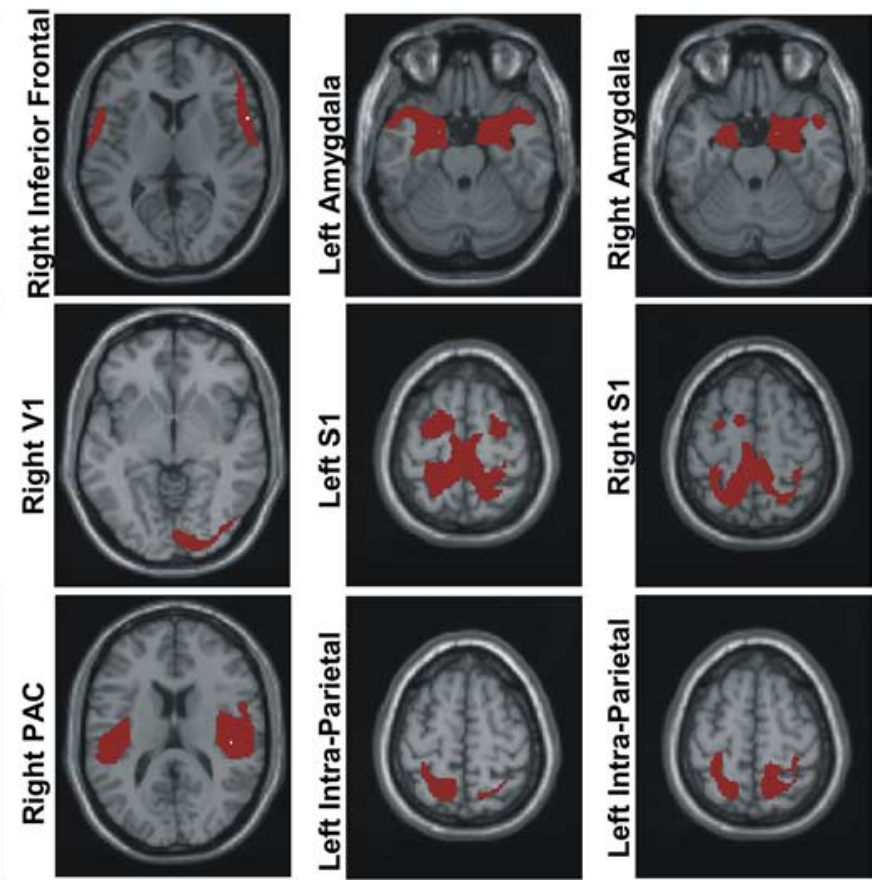

Figure 1. Positive associations between our 12 regions of interest and the remaining voxels ( $p<0.05$ corrected).

amygdala expressed differential positive associations for male and female individuals ( $p<0.05$ corrected) (Fig. 3). Specifically, this region was positively associated with the bilateral anterior inferior temporal cortex in males more than in females (left, $x=$ $-32, y=5, z=-39, \mathrm{Z}$-score $=4.5$; right, $x=65, y=-15, z=$ $-29, \mathrm{Z}$-score $=5.7)$ and with the right angular gyrus in females more than males $(x=47, y=-53, z=39$; Z-score $=4.5)$. This double dissociation suggests that, despite the overall consistency between male and female groups, associations between different regions of the human brain are modulated by gender.

Inspection of the gender-specific correlations revealed that the increased association between left amygdala and left anterior inferior temporal cortex in males $>$ females was attributable to a strong positive association in males (Z-score $=8.0 ; p<0.05$ corrected) and only a positive trend in females $(\mathrm{Z}$-score $=3.1$; $p<0.001$ uncorrected). In contrast, increased association between left amygdala and right anterior inferior temporal cortex in males $>$ females was attributable to a positive trend in males (Z-score $=3.9 ; p<0.001$ uncorrected) and a negative trend in females (Z-score $=3.6 ; p<0.001$ uncorrected). Finally, the increased association between left amygdala and right angular gyrus in females $>$ males was attributable to a strong positive trend in females $(Z$-score $=4.1 ; p<0.001$ uncorrected) and a negative trend in males (Z-score $=1.1)$. Inspection of gender-specific regional densities also revealed that the density of left amygdala was larger in males than in females (Fig. 3). This observation was consistent with previous investigations of gender-related brain asymmetries using similar techniques (Good et al., 2001).

\section{Discussion}

In this study, we demonstrate for the first time that the gray matter densities of different regions of the human cortex covary positively and negatively across healthy individuals. Positive associations were detected between homotopic regions, between heterotopic regions, and between ipsilateral regions. In contrast, negative associations were detected only between heterotopic re- 
Table 2. Negative, symmetric associations between our regions of interest and the other areas of the human cortex

\begin{tabular}{|c|c|c|c|c|}
\hline & $x$ & $y$ & $z$ & Z-score \\
\hline \multicolumn{5}{|l|}{$\begin{array}{l}\text { Left inferior frontal, pars triangularis } \\
\text { Ipsilateral effects }\end{array}$} \\
\hline Left hippocampus & -26 & -18 & -11 & 5.3 \\
\hline \multicolumn{5}{|l|}{$\begin{array}{l}\text { Right inferior frontal, pars triangularis } \\
\text { Ipsilateral effects }\end{array}$} \\
\hline Right hippocampus & 27 & -27 & -18 & 4.8 \\
\hline \multicolumn{5}{|l|}{$\begin{array}{l}\text { Left primary visual cortex } \\
\text { NS }\end{array}$} \\
\hline \multicolumn{5}{|l|}{ Right primary visual cortex } \\
\hline \multicolumn{5}{|l|}{ Left primary auditory cortex } \\
\hline $\begin{array}{l}\text { Right primary auditory cortex } \\
\text { NS }\end{array}$ & & & & \\
\hline \multicolumn{5}{|l|}{$\begin{array}{l}\text { Left amygdala } \\
\text { |psilateral effects }\end{array}$} \\
\hline Left supramarginal gyrus & -62 & -38 & 39 & 5.9 \\
\hline \multicolumn{5}{|l|}{ Heterotopic effects } \\
\hline Right supramarginal gyrus & 60 & -36 & 41 & 5.1 \\
\hline $\begin{array}{l}\text { Right amygdala } \\
\text { Ipsilateral effects }\end{array}$ & & & & \\
\hline Right supramarginal gyrus & 59 & -36 & 44 & 4.5 \\
\hline \multicolumn{5}{|l|}{ Heterotopic effects } \\
\hline Left supramarginal gyrus & -60 & -33 & 41 & 4.7 \\
\hline \multicolumn{5}{|l|}{ Left S1 } \\
\hline \multicolumn{5}{|l|}{ Ipsilateral effects } \\
\hline Superior frontopolar gyrus & -9 & 65 & 0 & 4.4 \\
\hline Left inferior anterior temporal & -29 & 2 & -45 & 4.6 \\
\hline Left cerebellum & -41 & -68 & -47 & 5.3 \\
\hline \multicolumn{5}{|l|}{ Heterotopic effects } \\
\hline Superior frontopolar gyrus & 9 & 65 & -2 & 4.9 \\
\hline Right inferior anterior temporal & 27 & 8 & -39 & 6.6 \\
\hline Right cerebellum & 42 & -68 & -48 & 5.5 \\
\hline \multicolumn{5}{|l|}{ Right S1 } \\
\hline Superior frontopolar gyrus & 8 & 62 & -11 & 6.3 \\
\hline Right inferior anterior temporal & 26 & 6 & -38 & 5.1 \\
\hline Right cerebellum & 44 & -74 & -47 & 5.9 \\
\hline \multicolumn{5}{|l|}{ Heterotopic effects } \\
\hline Superior frontopolar gyrus & -5 & 62 & -11 & 5.8 \\
\hline Left inferior anterior temporal & -32 & 2 & -44 & 4.6 \\
\hline Left cerebellum & -42 & -69 & -48 & 5.7 \\
\hline \multicolumn{5}{|l|}{$\begin{array}{l}\text { Left intraparietal } \\
\text { NS }\end{array}$} \\
\hline $\begin{array}{l}\text { Right intraparietal } \\
\text { NS }\end{array}$ & & & & \\
\hline
\end{tabular}

AllZ-scores are significant at $p<0.05$ (corrected). These effects are consistent for males (M) and females (F). NS, Not significant.

gions and between ipsilateral regions. Critically, these findings cannot be explained by overall brain size, because we included total gray matter density as a confounding variable in the analysis. Furthermore, these effects cannot be induced by the $12 \mathrm{~mm}$ smoothing kernel used during preprocessing (supplemental data, available at www.jneurosci.org as supplemental material).

We report that the density of a region is generally a good predictor of the density of the homotopic region, with the striking exception of visual cortex. The "dissociation" between left and right visual cortex appears to be specific to V1, because strong positive associations were found between left and right extrastriate regions. We note that $\mathrm{V} 1$ is the only region of the macaque brain that expresses very limited interhemispheric connectivity. Specifically, callosal connections in this region are sparse and
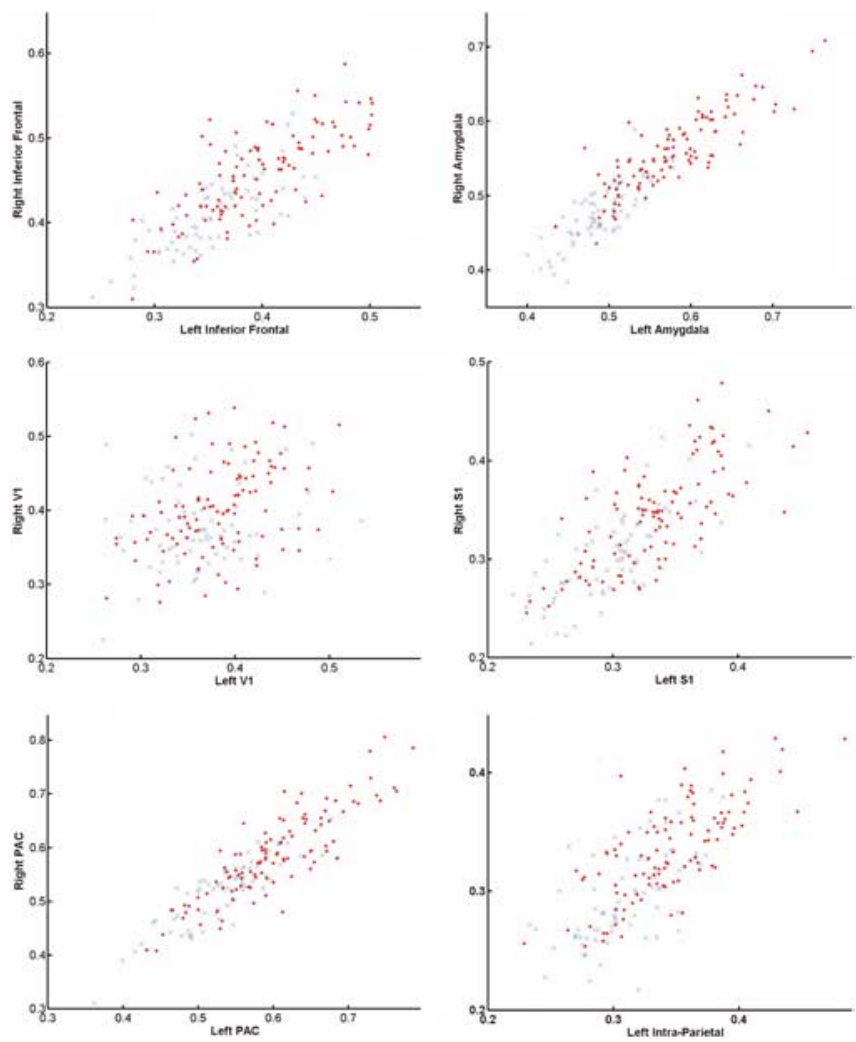

Figure 2. Gray matter correlations between homotopic regions, measured as cubic millimeters of gray matter per voxel, for males (red dots) and females (blues crosses). The $x$-and $y$-axes report gray matter volume for left- and right-lateralized regions, respectively. It can be seen that homotopic regions typically covary in volume, with the exception of V1. PAC, Primary auditory cortex.

restricted to a very narrow band in proximity of the V1/V2 border (Lund et al., 1975; Kennedy et al., 1986; Clarke and Miklossy, 1990; Olavarria and Abel, 1996; Abel et al., 2000). Conversely, extrastriate regions in the macaque brain exhibit an extensive and robust pattern of callosal connections that link loci located at the same distance from the interhemispheric fissure (Zeki, 1970; van Essen and Zeki, 1978; Kennedy et al., 1986; Clarke and Miklossy, 1990; Olavarria and Abel, 1996; Abel et al., 2000). Thus, the pattern of associations found in our study parallels the pattern of callosal connections found in the macaque brain. This finding suggests that the development of positive associations between homotopic regions may be critically dependent on mutually trophic influences that are mediated by callosal connections (Caviness and Takahashi, 1995; Ferrer et al., 1995; Herrup and Busser, 1995).

We also found that a number of homotopic regions express hemispheric differences in the patterns of regional covariance. For instance, "lateralized" associations were found in inferior frontal cortex, somatosensory cortex, and intraparietal cortex. Previous neuroanatomical studies have reported structural asymmetries in several regions of the human cortex, including frontal, temporal, and occipital areas (for review, see Good et al., 2001). Furthermore, neuropsychological and neuroimaging studies have provided evidence for hemispheric lateralization of brain function across several domains. For instance, lateralized brain responses have been reported for language (Frost et al., 1999), somatosensory (Coghill et al., 2001), affective (Morris et al., 1998), and autonomic (Yoon et al., 1997) processing. Our study demonstrates that hemispheric lateralization is expressed 
Table 3. Hemispheric asymmetries in the structural associations between our regions of interest and the other areas of the human cortex ( $p<0.05$ corrected)

\begin{tabular}{|c|c|c|c|c|c|c|c|c|}
\hline & $x$ & $y$ & $z$ & $\begin{array}{l}\text { Positive } \\
\text { associations }\end{array}$ & $\begin{array}{l}\text { Positive } \\
\text { associations }\end{array}$ & $\begin{array}{l}\text { Negative } \\
\text { associations }\end{array}$ & $\begin{array}{l}\text { Positive } \\
\text { associations }\end{array}$ & $\begin{array}{l}\text { Negative } \\
\text { associations }\end{array}$ \\
\hline & & & & $L$ versus $R$ & $\mathrm{~L}$ & $\mathrm{~L}$ & $\mathrm{R}$ & $\mathrm{R}$ \\
\hline \multicolumn{9}{|l|}{$\begin{array}{l}\text { Inferior frontal, pars triangularis } \\
\text { Ipsilateral effects } R>L\end{array}$} \\
\hline Inferior frontal, pars opercularis & 54 & 6 & 24 & 5.2 & 1.7 & & 6.8 & \\
\hline \multicolumn{9}{|l|}{ Primary visual cortex } \\
\hline \multicolumn{9}{|l|}{$\begin{array}{l}\text { Primary auditory cortex } \\
\text { NS }\end{array}$} \\
\hline \multicolumn{9}{|l|}{ Amygdala } \\
\hline \multicolumn{9}{|l|}{ S1 } \\
\hline \multicolumn{9}{|l|}{ Ipsilateral effects $L>R$} \\
\hline Superior frontal gyrus & -9 & 0 & 62 & 5.9 & 5.2 & & & 2.5 \\
\hline Parahippocampal gyrus & -15 & -35 & -6 & 4.9 & 6.8 & & 0.5 & \\
\hline $\begin{array}{c}\text { Paracentral lobule } \\
\text { Ipsilateral effects } R>L\end{array}$ & \multicolumn{7}{|c|}{ Ipsilateral effects $\mathrm{R}>\mathrm{L}$} & \\
\hline Superior frontopolar gyrus & 8 & 65 & 3 & 4.5 & & 5.9 & 0.1 & \\
\hline Cuneus & 15 & -89 & 20 & 4.5 & & 3.6 & 2.7 & \\
\hline \multicolumn{9}{|l|}{ Heterotopic effects $L>R$} \\
\hline Superior frontal gyrus & -6 & -6 & 54 & 4.6 & 4.1 & & & 1.9 \\
\hline Cerebellum & -20 & -53 & -60 & 5.0 & 5.6 & & & 0.7 \\
\hline \multicolumn{9}{|l|}{ Heterotopic effects $R>L$} \\
\hline Superior parietal gyrus & 21 & -60 & 63 & 7.8 & 7.4 & & 8.9 & \\
\hline Angular gyrus & 42 & -56 & 47 & 5.3 & 2.9 & & 7.7 & \\
\hline \multicolumn{9}{|l|}{ Intraparietal } \\
\hline \multicolumn{9}{|l|}{ Heterotopic effects $R>L$} \\
\hline Angular gyrus & 39 & -51 & 45 & 5.6 & 2.3 & & 7.2 & \\
\hline
\end{tabular}

Ipsilateral effects refer to associations between ipsilateral regions that differ between left $(\mathrm{L})$ and right $(\mathrm{R})$ hemispheres. Heterotopic effects refer to associations between heterotopic regions that differ between left and right hemispheres. " $\mathrm{L}>\mathrm{R}$ " indicates associations that are stronger when the "reference" region is left lateralized than when the reference region is right lateralized. " $\mathrm{R}>\mathrm{L}$ " indicates associations that are stronger when the reference region is right lateralized than when the reference region is left lateralized. "Positive associations $\mathrm{L}$ " and "Negative associations $\mathrm{L}$ " refer to the strength of positive and negative associations, respectively, when the reference region is left lateralized. "Positive associations R" and "Negative associations R" refer to the strength of positive and negative associations, respectively, when the reference region is right lateralized. Z-scores significant at $p<0.05$ (corrected) are reported in bold. NS, Not significant.

not only in terms of regional differences or lateralized brain responses but also in terms of asymmetric patterns of associations between regions.

The patterns of associations identified in our study were remarkably similar for male and female individuals. However, a gender-related double dissociation is found in the left amygdala, which is positively associated with bilateral anterior inferior temporal cortex in males and right angular gyrus in females. We note that this region typically expresses gender-related activation in studies of implicit memory for emotionally charged material. For example, activation of the left amygdala has been reported for long-term incidental memory of arousing material in male but not in female individuals (Cahill et al., 2001, 2004; Canli et al., 2002). We also note that both anterior inferior temporal cortex and angular gyrus are involved in various aspects of memory formation, maintenance, and retrieval (Wagner et al., 1998; Mecklinger et al., 2002; Weis et al., 2004). One could therefore speculate that gender-related brain responses in the left amygdala are mediated by the gender-related patterns of associations identified in our study.

Our findings are consistent with previous reports of significant covariation in the volume of circumscribed brain regions (Raz et al., 1997, 2004, 2005). However, there are a number of methodological and theoretical differences between our investigation and previous studies. Here, we used VBM to investigate gray matter density in terms of cubic millimeters of gray matter per voxel, whereas previous work used volumetric analysis to examine differences in regional volume. Although a comparison of the two methodologies has produced consistent (Keller et al.,
2004) as well as inconsistent (Tisserand et al., 2002) results, gray matter density and regional volume are different aspects of brain morphology. In addition VBM is a semiautomated technique that tests for significant effects across all voxels of the brain, whereas previous studies tested for the correlations between a priori regions of interest. Finally, the aim of our study was to use intersubject variability to characterize structural covariance in the human cortex. We therefore used age as a confounding variable to discard any pattern of covariance that could be explained by regionally specific, age-related decline. In contrast, the objective of previous work was to characterize the differential effect of aging on different regions of the brain. In these studies, correlations were calculated across a broad age range and were dependent on regionally specific, age-related changes.

In the present investigation, regional densities were defined as cubic millimeters of gray matter per voxel and should not be confused with cell packing density measured cytoarchitectonically. It is currently unclear whether the differences in gray matter density typically observed with VBM are related to differences in neuropil, neuronal size, dendritic or axonal arborization. In the neocortex, increased gray matter volume could also result from more folding as well as thicker gray matter (Mechelli et al., 2005). The biological significance of gray matter density, as measured using MRI techniques, is of great interest given the growing number of VBM studies being published but can only be addressed by methods other than MRI. It should also be noted that VBM uses a relatively simple warping method that only attempts to register the brain images globally. As a result, significant effects may result not only from differences in gray matter density between exper- 

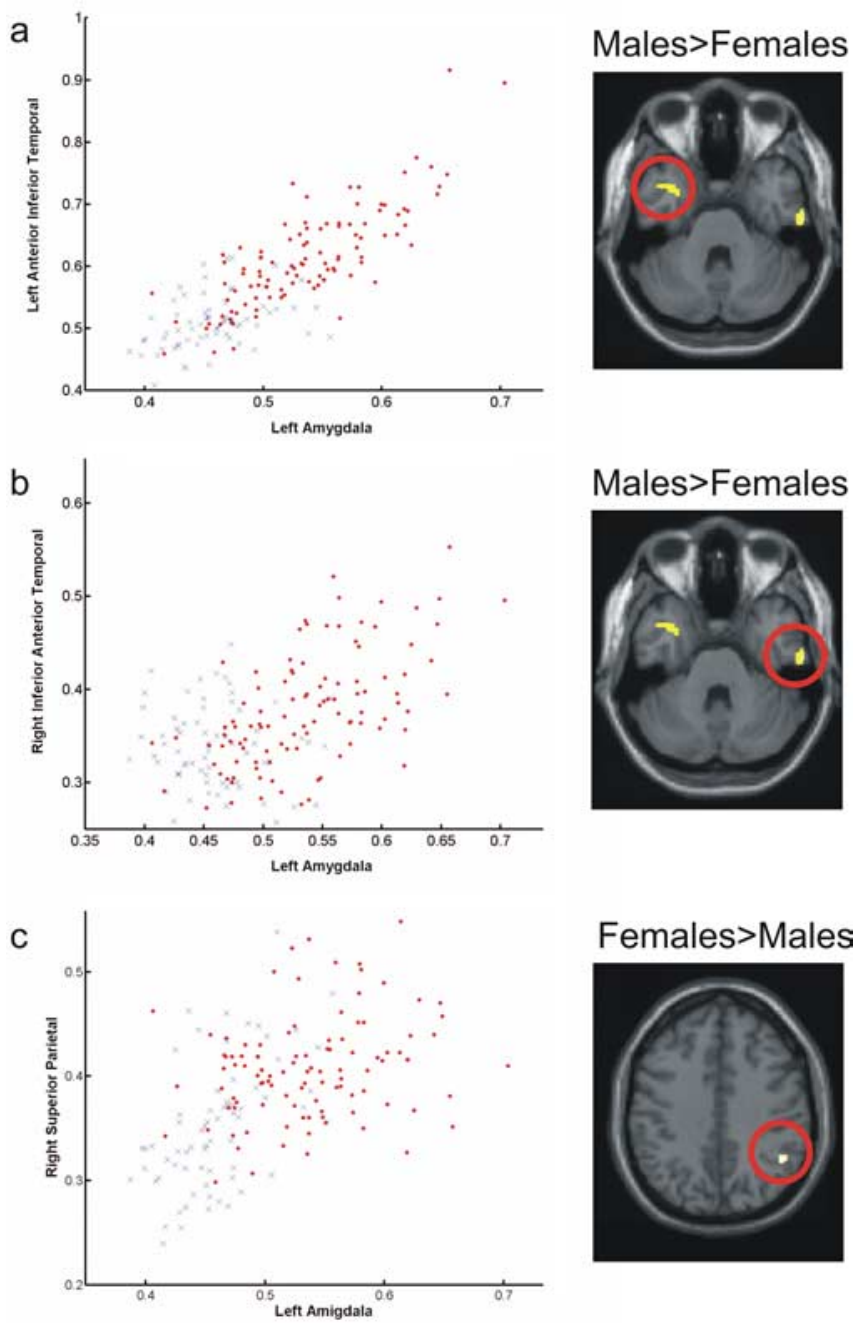

Figure 3. Gray matter correlations between left amygdala and left anterior inferior temporal cortex $(\boldsymbol{a})$, right anterior inferior temporal cortex $(\boldsymbol{b})$, and right angular gyrus $(\boldsymbol{c})$. The $x$ - and $y$-axes report regional densities, measured as cubic millimeters of gray matter per voxel, for males (red dots) and females (blues crosses). On the right of each correlation graph, regions $\boldsymbol{a}-\boldsymbol{c}$ are highlighted by the red circle.

imental groups but also from differences in the deployment of brain structures in one group relative to another (Ashburner and Friston, 2001; Mechelli et al., 2005). This means that the positive and negative correlations we report might be partly influenced by intersubject variance in the placement of our regions of interest. If this is the case, our effects would still reflect "structural covariance" across individuals, but this would be induced by the placement of gray matter as opposed to its density.

A question of interest is whether the regions found to be associated in the present investigation are also functionally related. For instance, one could hypothesize that regions that are positively associated may also be part of the same functional network. A clear-cut answer to this question may not be possible, because the functional relationships among different regions of the human cortex are yet to be fully characterized. However, we note that some positively associated regions in the present investigation were found to be coactivated in previous functional imaging studies. For instance, Tan et al. (2001) reported that reading Chinese characters was associated with activation in a distributed set of regions that include bilateral inferior frontal gyrus, postcentral gyrus, pars opercularis, and anterior superior temporal cortex. In our investigation, we found that the inferior frontal gyrus was positively associated with postcentral gyrus, pars opercularis, and anterior superior temporal cortex. The overlap between the two studies provides anecdotal support for the hypothesis that regions that are positively associated in terms of gray matter density may also be part of the same functional network.

In the present study, we investigated the structural covariance of the human cortex in 172 healthy individuals. However, the associations we detected may be disrupted in some patient populations, although the mean regional densities per se may not be affected. In the only example of this sort, Woodruff et al. (1997) demonstrated a dissociation between gray matter volumes of superior temporal and frontal cortex in schizophrenics relative to controls, although neither region individually differed in mean volume between the two groups. The approach we use here may therefore be useful to investigate patterns of associations in patients and controls or in different groups of patients.

Our study establishes that the gray matter density of different regions of the human cortex is coordinated within an individual. However, it is unclear whether this coordination reflects the contribution of heredity (Suddath et al., 1990; Steinmetz et al., 1994; Bartley et al., 1997; Tramo et al., 1998; Thompson et al., 2001) or environmental influences (Maguire et al., 2000; Gaser and Schlaug 2003; Draganski et al., 2004; Mechelli et al., 2004). It is likely that both genetic and nongenetic factors are involved in determining the positive and negative associations we report. A related question of interest is whether the allocation of neural circuitry in a given brain influences behavior. Comparisons across species suggest that behavior for which animals show particular ability are reflected in the amount of underlying circuitry (Johnson, 1980; Burda et al., 1990; Kaas 1993; Purves et al., 1996). Furthermore, studies of healthy human beings indicate that performance is a function of the allocation of neural circuitry (Mechelli et al., 2004). It is therefore possible that the coordinated variations we report across the human cortex are at least in part the basis for differences in individual behavior.

\section{References}

Abel PL, O’Brien BJ, Olavarria JF (2000) Organization of callosal linkages in visual area V2 of macaque monkey. J Comp Neurol 428:278-293.

Andrews TJ, Halpern SD, Purves D (1997) Correlated size variations in human visual cortex, lateral geniculate nucleus, and optic tract. J Neurosci 17:2859-2868.

Ashburner J, Friston KJ (2000) Voxel-based morphometry: the methods. NeuroImage 11:805-821.

Ashburner J, Friston KJ (2001) Why voxel-based morphometry should be used. NeuroImage 14:1238-1243.

Bartley AJ, Jones DW, Weinberger DR (1997) Genetic variability of human brain size and cortical gyral patterns. Brain 120:257-269.

Burda H, Burns V, Muller M (1990) Sensory adaptations in subterrean mammals. In: Evolution of subterrean mammals at the organismal and molecular levels (Nevo E, Reig OA, eds), pp 269-293. New York: Wiley.

Cahill L, Haier RJ, White NS, Fallon J, Kilpatrick L, Lawrence C, Potkin SG, Alkire MT (2001) Sex-related difference in amygdala activity during emotionally influenced memory storage. Neurobiol Learn Mem 75:1-9.

Cahill L, Uncapher M, Kilpatrick L, Alkire MT, Turner J, Cahill L, Uncapher M, Kilpatrick L, Alkire MT, Turner J (2004) Sex-related hemispheric lateralization of amygdala function in emotionally influenced memory: an FMRI investigation. Learn Mem 11:261-266.

Canli T, Desmond JE, Zhao Z, Gabrieli JD (2002) Sex differences in the neural basis of emotional memories. Proc Natl Acad Sci USA 99:10789-10794.

Caviness Jr VS, Takahashi T (1995) Proliferative events in the cerebral ventricular zone. Brain Dev 17:159-163.

Clarke S, Miklossy J (1990) Occipital cortex in man: organization of callosal connections, related myelo- and cytoarchitecture, and putative boundaries of functional visual areas. J Comp Neurol 298:188-214. 
Coghill RC, Gilron I, Iadarola MJ (2001) Hemispheric lateralization of somatosensory processing. J Neurophysiol 85:2602-2612.

Draganski B, Gaser C, Busch V, Schuierer G, Bogdahn U, May A (2004) Neuroplasticity: changes in gray matter induced by training. Nature 427:311-312.

Ferrer I, Blanco R, Carulla M, Condom M, Alcantara S, Olive M, Planas A (1995) Transforming growth factor-alpha immunoreactivity in the developing adult brain. Neuroscience 66:189-199.

Frost JA, Binder JR, Springer JA, Hammeke TA, Bellgowan PS, Rao SM, Cox RW (1999) Language processing is strongly left lateralized in both sexes. Evidence from functional MRI. Brain 122:199-208.

Gaser C, Schlaug G (2003) Brain structures differ between musicians and non-musicians. J Neurosci 23:9240-9245.

Good CD, Johnsrude I, Ashburner J, Henson RN, Friston KJ, Frackowiak RS (2001) Cerebral asymmetry and the effects of sex and handedness on brain structure: a voxel-based morphometric analysis of 465 normal adult human brains. NeuroImage 14:685-700.

Herrup K, Busser JC (1995) The induction of multiple cell cycle events precedes target-related neuronal death. Development 121:2385-2395.

Johnson JI (1980) Morphological correlates of specialized elaborations in somatic sensory cerebral cortex. In: Comparative neurology of the telencephalon (Ebesson SOE, ed), pp 423-447. New York: Plenum.

Kaas JH (1993) The organization of the visual cortex in primates: problems, conclusions and the use of comparative studies in understanding the human brain. In: Functional organization of the human visual cortex (Gulyas B, Ottson D, Roland PE, eds), pp 1-11. New York: Pergamon.

Keller SS, Wilke M, Wieshmann UC, Sluming VA, Roberts N (2004) Comparison of standard and optimized voxel-based morphometry for analysis of brain changes associated with temporal lobe epilepsy. NeuroImage 23:860-868.

Kennedy DN, Lange N, Makris N, Bates J, Mayer J, Caviness Jr VS (1998) Gyri of the human neocortex: an MRI-based analysis of volume and variance. Cereb Cortex 8:372-384.

Kennedy H, Dehay C, Bullier J (1986) Organization of callosal connections of visual areas V1 and V2 in the macaque monkey. J Comp Neurol 247:398-415.

Kleinbaum DG, Kupper LL, Muller KE (1988) Applied regression analysis and multivariable methods, pp 92-93. Belmont, CA: Duxbury.

Lund JS, Lund RD, Hendrickson AE, Bunt AH, Fuchs AF (1975) The origin of efferent pathways from the primary visual cortex, area 17 , of the macaque monkey as shown by retrograde transport of horseradish peroxidase. J Comp Neurol 164:287-303.

Maguire EA, Gadian DG, Johnsrude IS, Good CD, Ashburner J, Frackowiak RS, Frith CD (2000) Navigation-related structural change in the hippocampi of taxi drivers. Proc Natl Acad Sci USA 97:4398-4403.

Mechelli A, Crinion JT, Noppeney U, O’Doherty J, Ashburner J, Frackowiak RS, Price CJ (2004) Neurolinguistics: structural plasticity in the bilingual brain. Nature 431:757.

Mechelli A, Price CJ, Friston KJ, Ashburner J (2005) Voxel-based morphometry of the human brain: methods and applications. Curr Med Imaging Rev 1:105-113.

Mecklinger A, Gruenewald C, Besson M, Magnié M-N, Von Cramon DY (2002) Separable neuronal circuitries for manipulable and nonmanipulable objects in working memory. Cereb Cortex 12:1115-1123.

Morris JS, Ohman A, Dolan RJ (1998) Conscious and unconscious emotional learning in the human amygdala. Nature 393:467-470.

Olavarria JF, Abel PL (1996) The distribution of callosal connections correlates with the pattern of cytochrome oxidase stripes in visual area V2 of macaque monkeys. Cereb Cortex 6:631-639.
Purves, D, White LE, Zheng D, Andrews TJ, Riddle DR (1996) Brain size, behavior and the allocation of neural space. In: Individual development over the lifespan: biological and psychosocial perspectives (Magnusson D, ed), pp 162-178. Cambridge, UK: Cambridge UP.

Raz N, Gunning FM, Head D, Dupuis JH, McQuain JD, Briggs SD, Loken WJ, Thornton AE, Acker JD (1997) Selective aging of the human cerebral cortex observed in vivo: differential vulnerability of the prefrontal gray matter. Cereb Cortex 7:268-282.

Raz N, Gunning-Dixon F, Head D, Rodrigue KM, Williamson A, Acker JD (2004) Aging, sexual dimorphism, and hemispheric asymmetry of the cerebral cortex: replicability of regional differences in volume. Neurobiol Aging 25:377-396.

Raz N, Lindenberger U, Rodrigue KM, Kennedy KM, Head D, Williamson A, Dahle C, Gerstorf D, Acker JD (2005) Regional brain changes in aging healthy adults: general trends, individual differences and modifiers. Cereb Cortex, in press.

Sluming V, Barrick T, Howard M, Cezayirli E, Mayes A, Roberts N (2002) Voxel-based morphometry reveals increased gray matter density in Broca's area in male symphony orchestra musicians. NeuroImage $17: 1613-1622$.

Steinmetz H, Herzog A, Huang Y, Hacklander T (1994) Discordant brainsurface anatomy in monozygotic twins. N Engl J Med 331:951-952.

Stephan KE, Marshall JC, Friston KJ, Rowe JB, Ritzl A, Zilles K, Fink GR (2003) Lateralized cognitive processes and lateralized task control in the human brain. Science 301:384-386.

Suddath RL, Christison GW, Torrey EF, Casanova MF, Weinberger DR (1990) Anatomical abnormalities in the brains of monozygotic twins discordant for schizophrenia. N Engl J Med 322:789-794.

Tan LH, Feng CM, Fox PT, Gao JH (2001) An fMRI study with written Chinese. NeuroReport 12:83-88.

Thompson PM, Cannon TD, Narr KL, van Erp T, Poutanen VP, Huttunen M, Lonnqvist J, Standertskjold-Nordenstam CG, Kaprio J, Khaledy M, Dail R, Zoumalan CI, Toga AW (2001) Genetic influences on brain structure. Nat Neurosci 4:1253-1258.

Tisserand DJ, Pruessner JC, Sanz Arigita EJ, van Boxtel MPJ, Evans AC, Jolles J, Uylings HBM (2002) Regional frontal cortical volumes decrease differentially in aging: an MRI study to compare volumetric approaches and voxel-based morphometry. NeuroImage 17:657-669.

Tramo MJ, Loftus WC, Stukel TA, Green RL, Weaver JB, Gazzaniga MS (1998) Brain size, head size, and intelligence quotient in monozygotic twins. Neurology 50:1246-1252.

van Essen DC, Zeki SM (1978) The topographic organization of rhesus monkey prestriate cortex. J Physiol (Lond) 277:193-226.

Wagner AD, Schacter DL, Rotte M, Koutstaal W, Maril A, Dale AM, Rosen BR, Buckner RL (1998) Building memories: remembering and forgetting of verbal experiences as predicted by brain activity. Science 281:1188-1191.

Weis S, Klaver P, Reul J, Elger CE, Fernández G (2004) Temporal and cerebellar brain regions that support both declarative memory formation and retrieval. Cereb Cortex 14:256-267.

Woodruff PW, Wright IC, Shuriquie N, Russouw H, Rushe T, Howard RJ, Graves M, Bullmore ET, Murray RM (1997) Structural brain abnormalities in male schizophrenics reflect fronto-temporal dissociation. Psychol Med 27:1257-1266.

Yoon BW, Morillo CA, Cechetto DF, Hachinski V (1997) Cerebral hemispheric lateralization in cardiac autonomic control. Arch Neurol 54:741-744.

Zeki S (1970) Interhemispheric connections of prestriate cortex in monkey. Brain Res 19:63-75. 